\title{
Using Visual Guides to Reduce Virtual Reality Sickness in First-Person Shooter Games: Correlation Analysis
}

\author{
Kwang-Ho Seok ${ }^{1}$, PhD; YeolHo Kim ${ }^{1}$, MS; Wookho Son ${ }^{2}$, PhD; Yoon Sang Kim ${ }^{1}$, PhD \\ ${ }_{1}^{1}$ BioComputing Lab, Institute for Bio-engineering Application Technology, School of Computer Science and Engineering, Korea University of \\ Technology and Education, Cheonan-si, Republic of Korea \\ ${ }^{2}$ Electronics \& Telecommunications Research Institute, Daejeon, Republic of Korea
}

\section{Corresponding Author:}

Yoon Sang Kim, PhD

BioComputing Lab, Institute for Bio-engineering Application Technology, School of Computer Science and Engineering

Korea University of Technology and Education

1600, Chungjeol-ro, Byeongcheon-myeon, Dongnam-gu

Cheonan-si, 31253

Republic of Korea

Phone: 820415601496

Email: yoonsang@koreatech.ac.kr

\begin{abstract}
Background: The virtual reality (VR) content market is rapidly growing due to an increased supply of VR devices such as head-mounted displays (HMDs), whereas VR sickness (reported to occur while experiencing VR) remains an unsolved problem. The most widely used method of reducing VR sickness is the use of a rest frame that stabilizes the user's viewpoint by providing fixed visual stimuli in VR content (including video). However, the earth-fixed grid and natural independent visual background that are widely used as rest frames cannot maintain VR fidelity, as they reduce the immersion and the presence of the user. A visual guide is a visual element (eg, a crosshair of first-person shooter [FPS]) that induces a user's gaze movement within the VR content while maintaining VR fidelity, whereas there are no studies on the correlation of visual guide with VR sickness.
\end{abstract}

Objective: This study aimed to analyze the correlation between VR sickness and crosshair, which is widely used as a visual guide in FPS games.

Methods: Eight experimental scenarios were designed and evaluated, including having the visual guide on/off, the game controller on/off, and varying the size and position of the visual guide to determine the effect of visual guide on VR sickness.

Results: The results showed that VR sickness significantly decreased when visual guide was applied in an FPS game. In addition, VR sickness was lower when the visual guide was adjusted to $30 \%$ of the aspect ratio and positioned in the head-tracking direction.

Conclusions: The experimental results of this study indicate that the visual guide can achieve VR sickness reduction while maintaining user presence and immersion in the virtual environment. In other words, the use of a visual guide is expected to solve the existing limitation of distributing various types of content due to VR sickness.

(JMIR Serious Games 2021;9(3):e18020) doi: 10.2196/18020

\section{KEYWORDS}

virtual reality; motion sickness; VR sickness; visual guide; VR fidelity

\section{Introduction}

Recently, virtual reality (VR) content based on head-mounted display (HMD) has been expanded to various industrial fields such as sports, medical care, education, and social network. Moreover, such content has been used in video games. However, most users have experienced "VR sickness" while using HMD-based VR content. VR sickness has symptoms similar to motion sickness, including nausea, oculomotor discomfort, and disorientation caused while experiencing an HMD-based VR [1]. To investigate the causes of VR sickness, various theories are being studied in a cognitive science approach. The popular sensory conflict theory [1] proposes that VR sickness is induced by an inconsistency between the visual and the vestibular or proprioceptive senses. In particular, the vection that occurs during the VR experience is the biggest cause of sensory conflict [2-4]. Additionally, the postural instability theory posits that VR sickness is caused by changes in human balance [5]. 
Furthermore, VR sickness may also be induced by various individual characteristics, such as age, gender, prior user experience, concentration, medical history, mental rotation, perceptual style, and dominant eye [6].

Until now, various studies have only been partially successful in attempting to reduce HMD-induced VR sickness by focusing on the device and the content. A typical method focusing on the device is the optimization of the delay time caused by head movement tracking response, rendering, image transmission, and display response speed [7]. Studies have also been conducted to reduce VR sickness in device elements such as resolution, frame rate, viewing angle, binocular parallax, and flicker fusion frequency [8-13]. Recent studies have evaluated methods focusing on content. These methods investigated the use of dynamic blurring with retinal tracking [14], optical flow reduction of peripheral vision [15], field of view control [16], and viewpoint snapping [17].

However, there is a disadvantage that these dynamic blurring methods limit the user's experience. Therefore, in other studies, VR sickness has been reduced by adding fixed or dynamic visual stimuli regardless of the motion of objects in the content [18-20]. This visual stimulus includes a rest frame that serves as a reference frame designed to induce a user's effective spatial perception. In particular, VR sickness was reduced by applying a virtual human nose as a rest frame to the content [21]. However, this artificial rest frame could not maintain VR fidelity, as it reduced user presence and immersion and provided a strong sense of heterogeneity to the user. VR sickness has further been reduced using the earth-fixed grid or the natural independent visual background (IVB) with rest frames, whereas it did not increase the presence and immersion of the user $[22,23]$. Particularly, VR sickness in first-person shooter (FPS) games was reduced when cockpits were added to the IVB, while the VR fidelity of the users was disturbed [24].

To overcome the problem of IVB, we discuss another visual element applied to VR FPS games called the visual guide, which refers to a visual element that induces a user's gaze movement within VR content. To our knowledge, there is no study investigating the effect of visual guide on the reduction of VR sickness while maintaining VR fidelity in VR FPS games. Therefore, in this study, we investigated how VR sickness reduction is affected by visual guide in FPS games. To do this, we performed experiments on a VR FPS game consisting of eight scenarios, including visual guide on/off, game controller on/off, and varying the size and position of the visual guide.

\section{Methods}

\section{Visual Guide Design in VR FPS Games}

In this section, we describe the visual guide used in this study. In VR FPS games, crosshairs, character path indicators, and maps are used as visual guides to provide situational awareness for the user to spatially determine their position in a virtual environment. In this study, we used crosshair as a visual guide to induce a user's gaze movement while maintaining VR fidelity in a space-environment FPS game. Crosshair is a 2D image composed of color, shape, line thickness, depth, size, and position elements. Figure 1 shows an example of crosshair used in the VR FPS game. The brilliant color, complex shape, bold line, and depth of a visual guide can reduce the presence and immersion of the user by increasing the visual stimulation [18]. Therefore, these elements are fixed, and they are designed as a white, circle, $1.0 \mathrm{px}$, and 0 , respectively by referring to commercial VR FPS games. Table 1 shows various crosshair in commercial VR FPS games.

Figure 1. Example of crosshair used in the virtual reality first-person shooter game. HMD: head-mounted display; SSQ: Simulator Sickness Questionnaire.

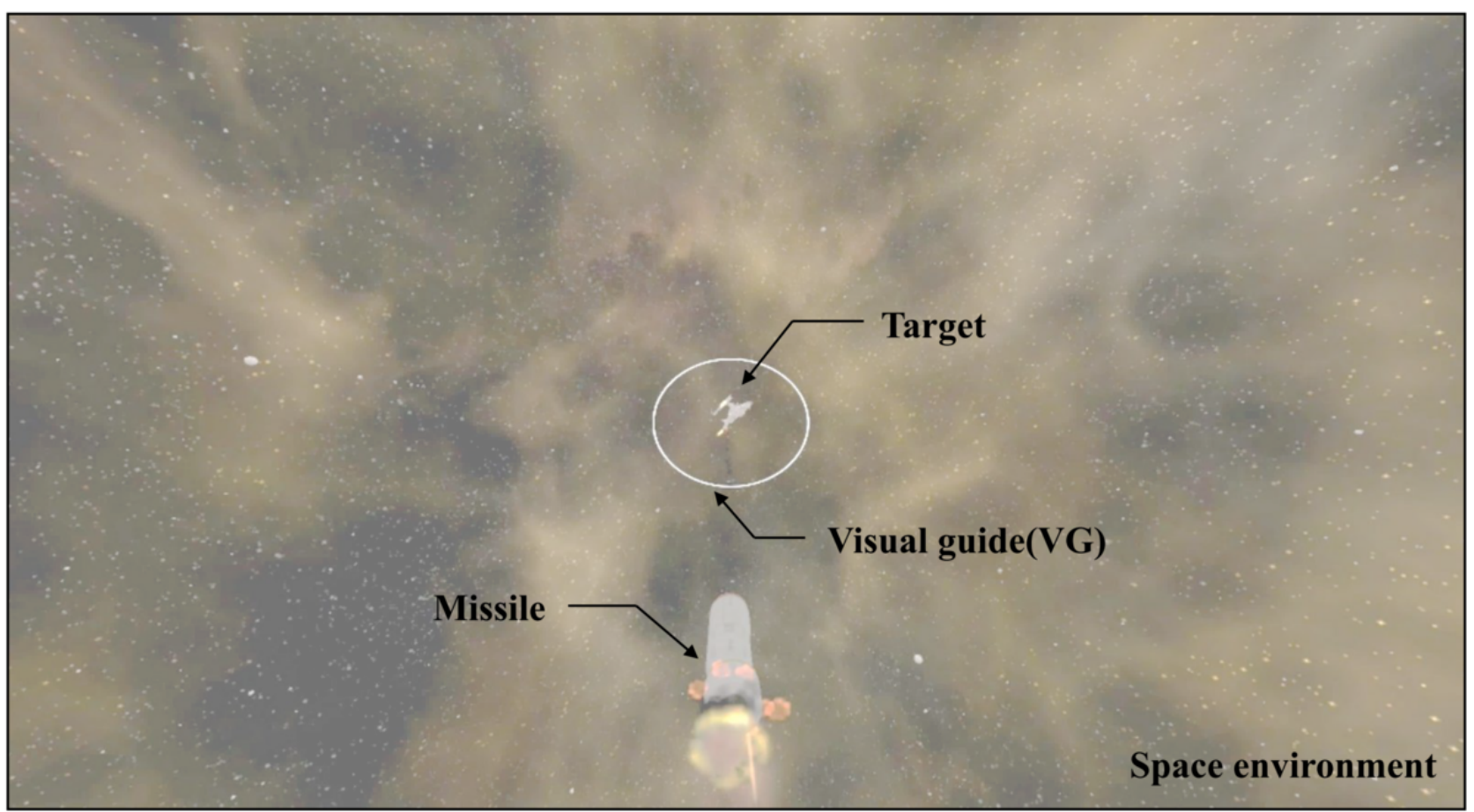


Table 1. Various crosshair in commercial virtual reality (VR) first-person shooter (FPS) games.

\begin{tabular}{|c|c|c|}
\hline No. & Name & Crosshair image \\
\hline 1 & Super Stardust Ultra VR & \\
\hline 2 & End Space VR & \\
\hline 3 & Elite: Dangerous & \\
\hline 4 & Gunjack & \\
\hline 5 & Sublevel Zero & \\
\hline
\end{tabular}
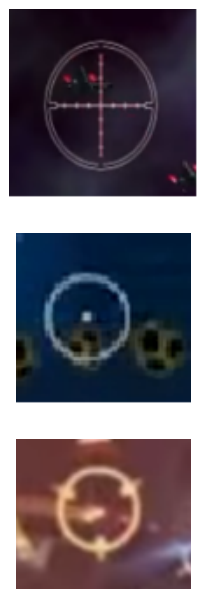

The size and position of the visual guide is considered a variable for VR sickness measurements. Therefore, we designed it as a variable. First, the size was designed to be $0 \%, 10 \%, 30 \%$, and $50 \%$ of the size of the aspect ratio (with $0 \%$ implying that there is no visual guide). Second, the position was synchronized to "none," "head-tracking direction with HMD (H)," "movement direction with game controller (G)," and "head-tracking direction with HMD and movement direction with game controller (H \& G)." That is, if the position is "none," then there is no visual guide. If the position is " $\mathrm{H}$," then the position of the visual guide is synchronized with the head-tracking direction by the HMD operation. If the position is "G," then the position of the visual guide is synchronized in the direction of forwarding movement, pitch, yaw, and roll rotation by the game controller operation. Finally, if the position is " $\mathrm{H} \& \mathrm{G}$," then the position of the visual guide is synchronized with the head-tracking direction by the HMD operation and the direction of forwarding movement, pitch, yaw, and roll by the game controller operation. 


\section{Experiments}

In this section, we describe the experimental environment and methods used to examine the effect of visual guide on VR sickness. The experimental environment consisted of the participant, experiment device, and experimental content.

We used a paired $t$ test method to analyze the results. This requires the experimental results to approximate the normal distribution. Therefore, the minimum sample size was set to 30 to satisfy a central limit theorem [25]. Eventually, 32 individuals (male: 23, female: 9) participated in the experiment.

All the participants were in their twenties without any HMD VR experience, and none had a medical history of hearing or balancing disorder. We received an institutional review board approval for testing of VR sickness by KOREATECH.

Before performing the experiment, each participant was administered a preliminary questionnaire (M1) comprising the Simulator sickness Questionnaire (SSQ) items, such as measurement, reliability, validity, score interpretation, etc [26,27], to evaluate their current motion sickness state. After the SSQ (M1) was completed, the participant wore the HMD, and the experiment was initiated.

The experimental equipment used was a head-tracking interface device HTC VIVE HMD and an Xbox One Wireless Controller. With these devices, the participants played the VR FPS game and answered the SSQ. The game controller has several functionalities including forward movement; rotation based on pitch, yaw and roll axes; missile launch targeting; and selection of answers in the SSQ. Experimental content is a VR FPS game in space environment because it easily causes VR sickness due to multiaxis movement. The user uses the HMD and the game controller operations to trace the enemy target and score it with the missile.

The experimental protocol consisted of preliminary experiment (M2), eight experimental scenarios (S1-S8), SSQ, and rest videos. Figure 2 shows the experimental protocol for VR sickness measurement. In the preliminary experiment (M2), the participants were taught how to operate the HMD and the game controller. The eight experimental scenarios were designed to measure VR sickness. If a participant is exposed to VR content for a long time, the VR sickness level will potentially increase. [28] Therefore, each scenario was composed of content of 60 $\mathrm{s}$ for the safety of the participants. These eight experimental scenarios were designed to have the visual guide on/off, the game controller on/off, and to vary sizes and positions of the visual guide to determine its effect on VR sickness. Table 2 shows the features of these eight scenarios.

In addition, the experimental scenarios were randomly placed and used to ensure reliability. For $240 \mathrm{~s}$ after each experimental scenario (including M2) ended, participants entered SSQ and watched the rest video to relax VR sickness. All participants were equally exposed to this rest video. When the experiment was completed, the SSQ data for the eight scenarios were automatically saved. From the VR sickness measurement experiment, preexperimental SSQ data (M1) and postexperimental SSQ data (S1-S8) were collected for each participant.

Figure 2. Experimental protocol for virtual reality sickness measurement. HMD: head-mounted display; SSQ: Simulator Sickness Questionnaire.

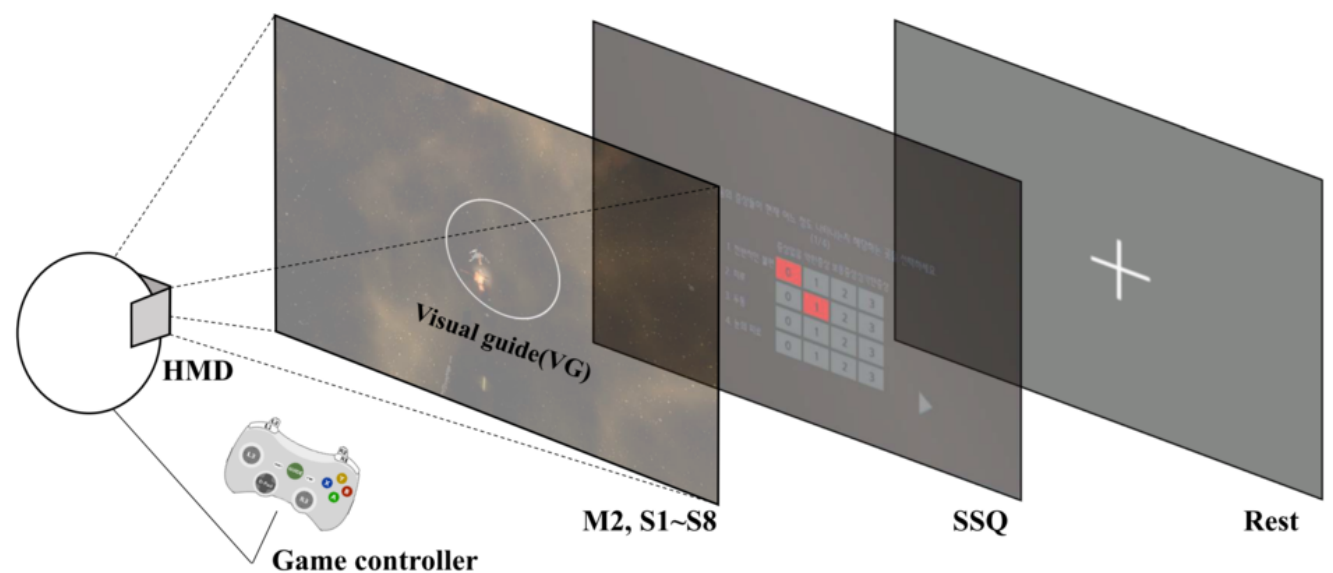

SSQ \& Rest

\begin{tabular}{|c|c|c|c|c|c|c|c|c|c|c|c|c|c|c|c|c|c|}
\hline & $i$ & & $i$ & & $i$ & & $i$ & & $i$ & & $i$ & & $i$ & & $i$ & & $i$ \\
\hline $120 \mathrm{~s}$ & $240 \mathrm{~s}$ & $60 \mathrm{~s}$ & $240 \mathrm{~s}$ & $60 \mathrm{~s}$ & $240 \mathrm{~s}$ & $60 \mathrm{~s}$ & $240 \mathrm{~s}$ & $60 \mathrm{~s}$ & $240 \mathrm{~s}$ & $60 \mathrm{~s}$ & $240 \mathrm{~s}$ & $60 \mathrm{~s}$ & $240 \mathrm{~s}$ & $60 \mathrm{~s}$ & $240 \mathrm{~s}$ & $60 \mathrm{~s}$ & $240 \mathrm{~s}$ \\
\hline M2 & & S1 & & S2 & & S3 & & S4 & & S5 & & S6 & & S7 & & S8 & \\
\hline
\end{tabular}


Table 2. Features of eight scenarios used in the experiment.

\begin{tabular}{|c|c|c|c|c|c|c|c|c|}
\hline Feature & S1 & S2 & S3 & $\mathrm{S} 4$ & S5 & S6 & S7 & S8 \\
\hline Game controller & Off & On & On & On & On & On & On & On \\
\hline Visual guide size $(\%)$ & 0 & 0 & 0 & 10 & 30 & 50 & 30 & 30 \\
\hline Visual guide position & None & None & None & $\mathrm{H}^{\mathrm{a}}$ & $\mathrm{H}$ & $\mathrm{H}$ & $\mathrm{G}^{\mathrm{b}}$ & $H \& G^{c}$ \\
\hline
\end{tabular}

${ }^{\mathrm{a}} \mathrm{H}$ : head-tracking direction with head-mounted display.

${ }^{\mathrm{b}} \mathrm{G}$ : movement direction with game controller.

${ }^{\mathrm{c}} \mathrm{H} \& \mathrm{G}$ : head-tracking direction with head-mounted display and movement direction with game controller.

Using these experimental protocols, we conducted two experiments to examine the effect of the visual guide on VR sickness. Experiment I investigated the effect of visual guide on/off on VR sickness. In the first step of experiment I, we investigated the effect of VR sickness when the visual guide is on/off with the game controller off. Thus, the SSQ data of S1 and S2 were used for the comparison of VR sickness. Figure 3 shows the S1 and S2 used in the first step of experiment I.

In the second step of experiment I, we investigated the effect of visual guide on/off with the game controller on. Thus, the SSQ data of S3 and S5 were used for the comparison of VR sickness. Figure 4 shows the S3 and S5 used in the second step of experiment I.
Experiment II investigated the effects of the visual guide's size and position on VR sickness. In the first step of experiment II, we investigated the effect of the visual guide's size with the game controller on. Thus, the SSQ data of S4, S5, and S6 and the SSQ data of S3 were used for the comparison of VR sickness. Figure 5 shows the S3, S4, S5, and S6 used in the first step of experiment II.

In the second step of experiment II, we investigated the effect of the visual guide's position with the game controller on. Thus, the SSQ data of S5, S7, and S8 using a visual guide with different positions at $30 \%$ of the size of the aspect ratio and the SSQ data of S3 were used for comparison of VR sickness. Figure 6 shows the shows S3, S5, S7, and S8 used in the second step of experiment II.

Figure 3. S1 and S2 used in the first step of experiment I. VG: visual guide.

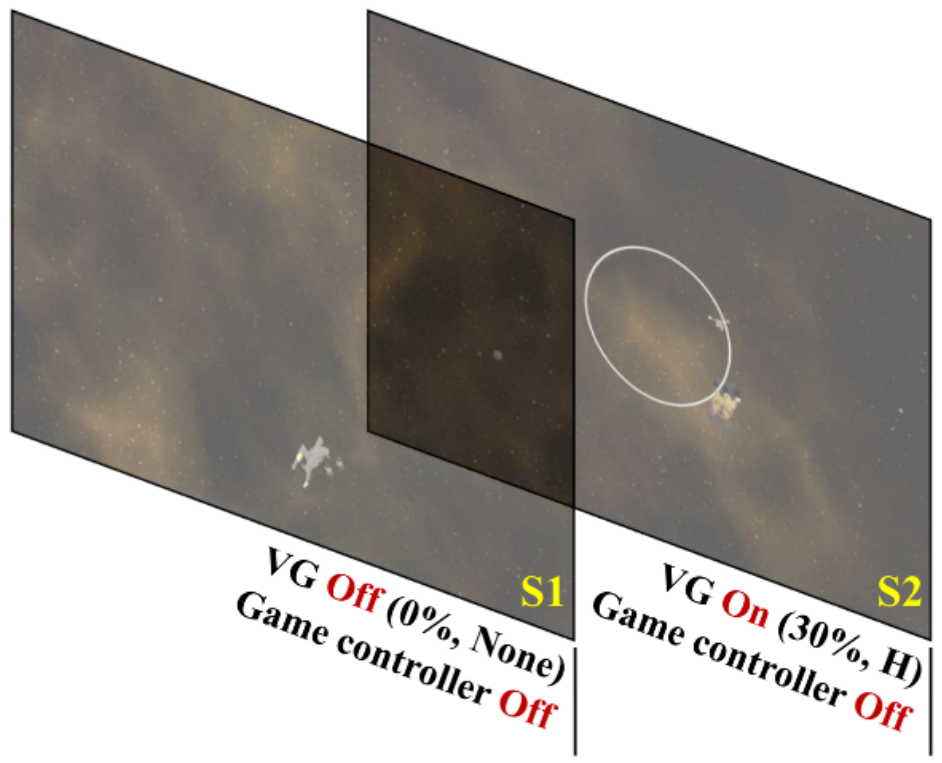


Figure 4. S3 and S5 used in the second step of experiment I. VG: visual guide.

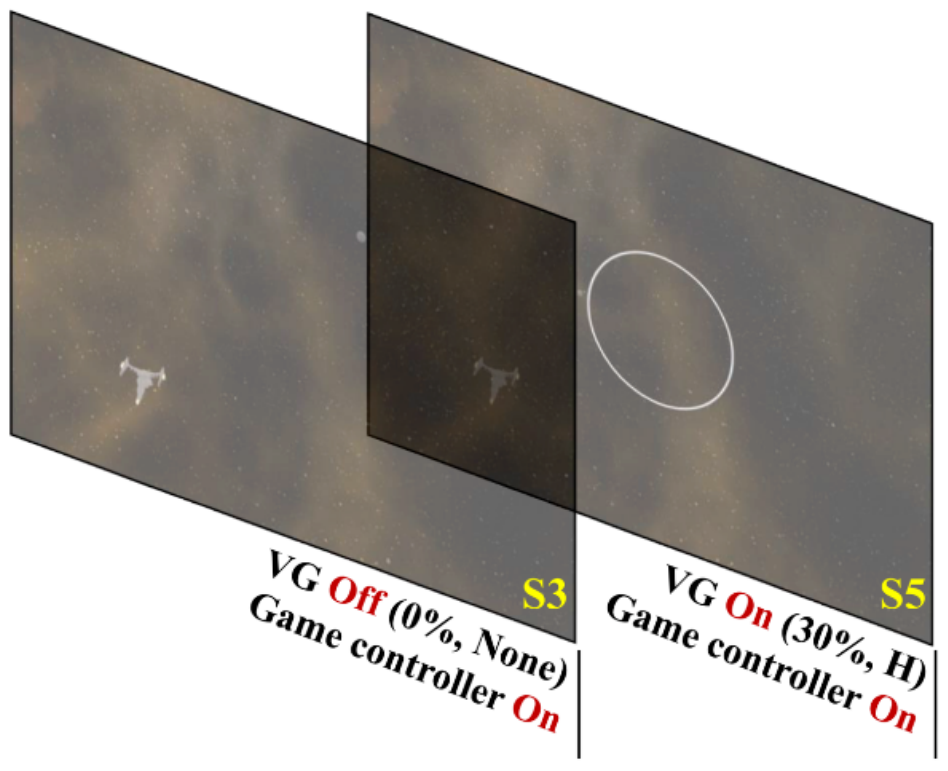

Figure 5. S3, S4, S5, and S6 used in the first step of experiment II. VG: visual guide.

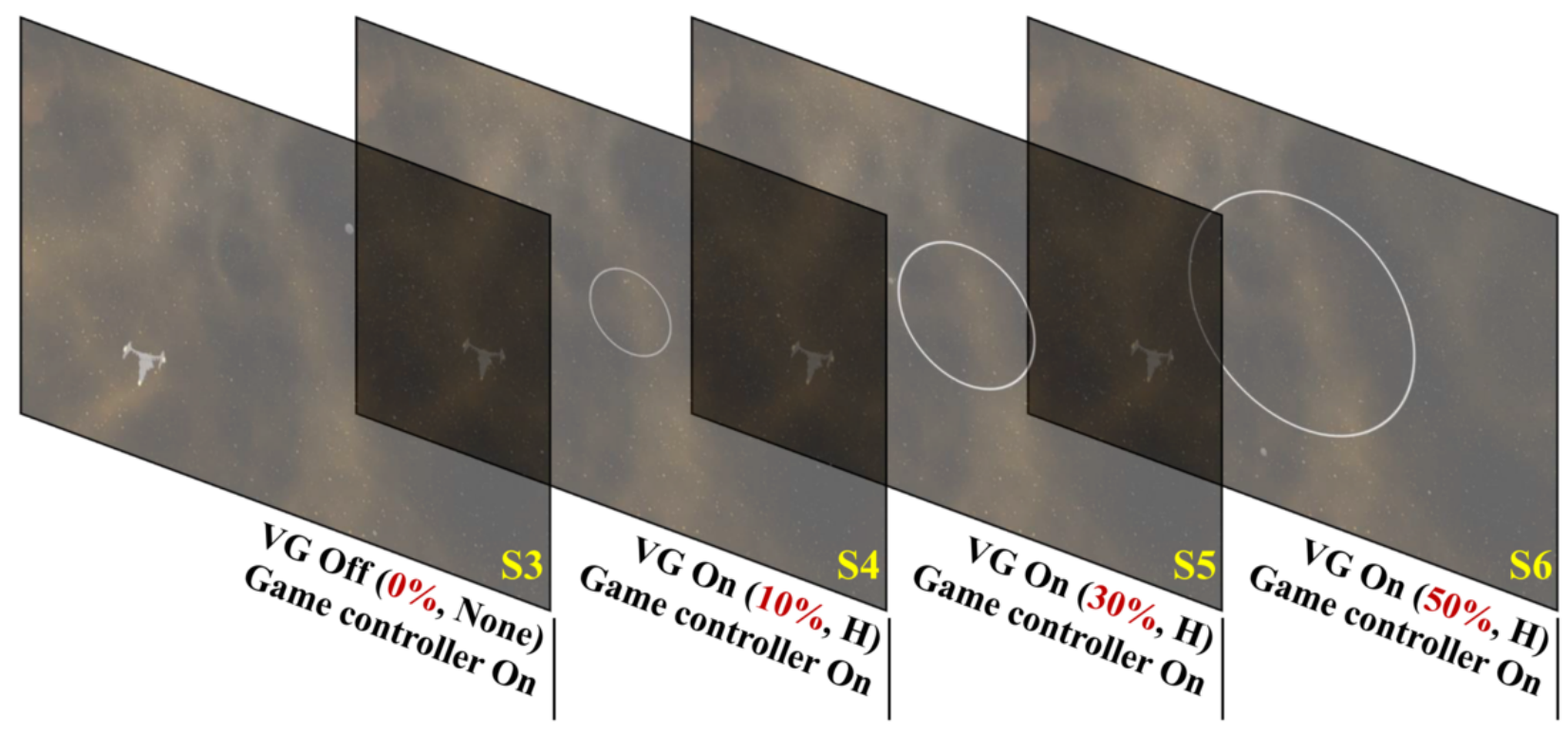


Figure 6. S3, S5, S7, and S8 used in the second step of Experiment II. VG: visual guide.

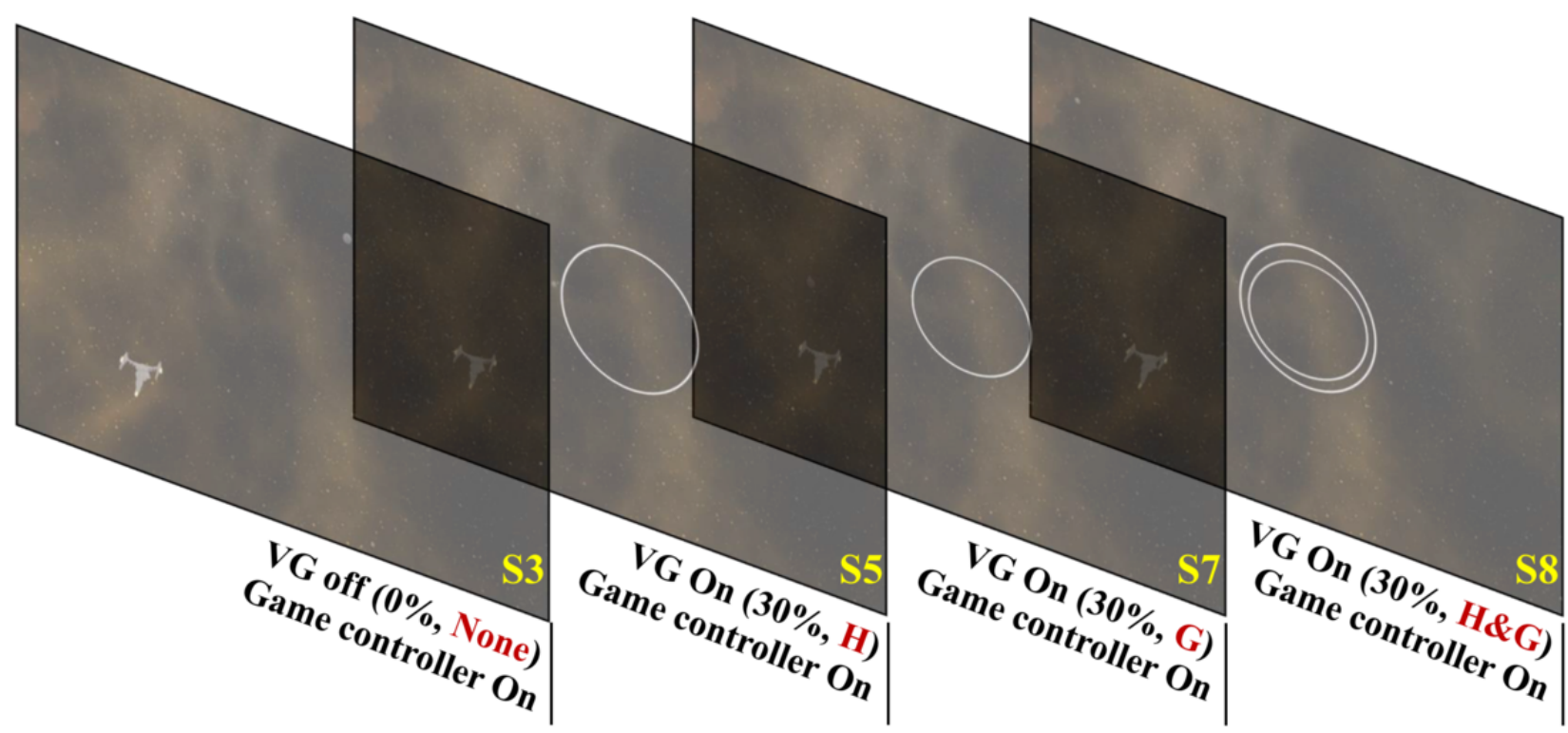

\section{Results}

\section{Overview}

First, we compared the SSQ preexperiment (M1) and postexperiment (S1-S8) data to verify that the experimental protocol for VR sickness measurement was well designed. From the paired $t$ test results, a significant difference was observed in VR sickness between M1 and S1-S8. Significantly higher nausea and disorientation symptoms were noted in S1-S8 than in M1. Hence, we confirmed that the participants had experienced nausea and disorientation. In addition, oculomotor discomfort in S1-S8 did not significantly increase because many participants had eye fatigue from M1. From the different SSQ values before and after VR exposure, the increase in the values of nausea and disorientation symptoms of the participants was confirmed. It was concluded that the scenarios for the VR sickness experiment were appropriately produced.

\section{Experiment I: Effects of Visual Guide On/Off}

In the first step of experiment $\mathrm{I}$, the results of the paired $t$ test showed no significant difference in VR sickness with respect to visual guide on/off when game controller was off; there was a negligible difference in nausea, oculomotor discomfort, disorientation, and total score values. In other words, there was no effect of visual guide with game controller off (watching video) on VR sickness. Table 3 shows the results of the paired $t$ test of visual guide on/off with game controller off. Figure 7 shows the results of the first step of experiment I (ie, difference in VR sickness with respect to visual guide on/off with game controller off).

In the second step of Experiment I, the paired $t$ test showed a significant difference in VR sickness with visual guide on/off and game controller on: nausea and total score values were significantly decreased, whereas oculomotor discomfort and disorientation values had no significant differences. Table 4 shows the results of the paired $t$ test of visual guide on/off with the game controller on. Figure 8 shows the results of the second step of Experiment I (difference in VR sickness of visual guide on/off with game controller on).

Table 3. Results of the paired $t$ test of the visual guide on/off with game controller off.

\begin{tabular}{|c|c|c|c|c|c|c|c|c|c|c|c|c|}
\hline \multirow[t]{3}{*}{ Scenario (state) } & \multicolumn{12}{|c|}{ Simulator Sickness Questionnaire } \\
\hline & \multicolumn{3}{|c|}{ Nausea } & \multicolumn{3}{|c|}{ Oculomotor discomfort } & \multicolumn{3}{|c|}{ Disorientation } & \multicolumn{3}{|c|}{ Total score } \\
\hline & Score & $t(d f)$ & $P$ value & Score & $t(d f)$ & $P$ value & Score & $t(d f)$ & $P$ value & Score & $t(d f)$ & $P$ value \\
\hline S1 (visual guide off) & 8.65 & $\mathrm{~N} / \mathrm{A}^{\mathrm{a}}$ & $\mathrm{N} / \mathrm{A}$ & 12.08 & N/A & N/A & 17.84 & N/A & $\mathrm{N} / \mathrm{A}$ & 14.14 & N/A & $\mathrm{N} / \mathrm{A}$ \\
\hline S2 (visual guide on) & 8.65 & $0(31)$ & $>.99$ & 13.50 & $-.649(31)$ & .52 & 16.97 & $.360(31)$ & .72 & 14.61 & $-.235(31)$ & .82 \\
\hline
\end{tabular}

${ }^{\mathrm{a}} \mathrm{N} / \mathrm{A}$ : not applicable. 
Figure 7. Results of the first step of experiment I (difference in virtual reality sickness with respect to visual guide on/off with game controller off). SSQ: Simulator Sickness Questionnaire; VG: visual guide.

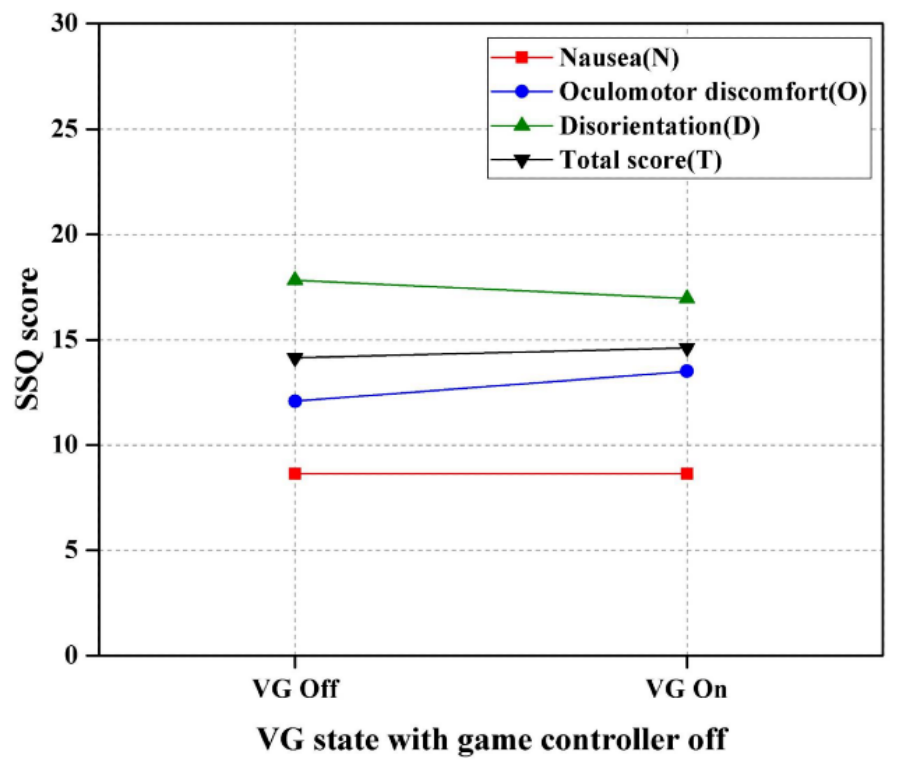

Table 4. Results of the paired $t$ test of the visual guide on/off with game controller on.

\begin{tabular}{|c|c|c|c|c|c|c|c|c|c|c|c|c|}
\hline \multirow[t]{3}{*}{ Scenario (state) } & \multicolumn{12}{|c|}{ Simulator Sickness Questionnaire } \\
\hline & \multicolumn{3}{|c|}{ Nausea } & \multicolumn{3}{|c|}{ Oculomotor discomfort } & \multicolumn{3}{|c|}{ Disorientation } & \multicolumn{3}{|c|}{ Total score } \\
\hline & Score & $t(d f)$ & $P$ value & Score & $t(d f)$ & $P$ value & Score & $t(d f)$ & $P$ value & Score & $t(d f)$ & $P$ value \\
\hline S3 (visual guide off) & 9.24 & $\mathrm{~N} / \mathrm{A}^{\mathrm{a}}$ & N/A & 15.87 & N/A & N/A & 20.45 & N/A & N/A & 16.95 & N/A & N/A \\
\hline S5 (visual guide on) & 5.66 & $2.547(31)$ & $.02^{\mathrm{b}}$ & 13.03 & $1.359(31)$ & .18 & 14.79 & $1.815(31)$ & .08 & 12.62 & $2.183(31)$ & .04 \\
\hline
\end{tabular}

${ }^{\mathrm{a}} \mathrm{N} / \mathrm{A}$ : not applicable.

${ }^{\mathrm{b}}$ Italicized values indicate statistical significance.

Figure 8. Results of the second step of experiment I (difference in virtual reality sickness of visual guide on/off with game controller on). SSQ: Simulator Sickness Questionnaire; VG: visual guide.

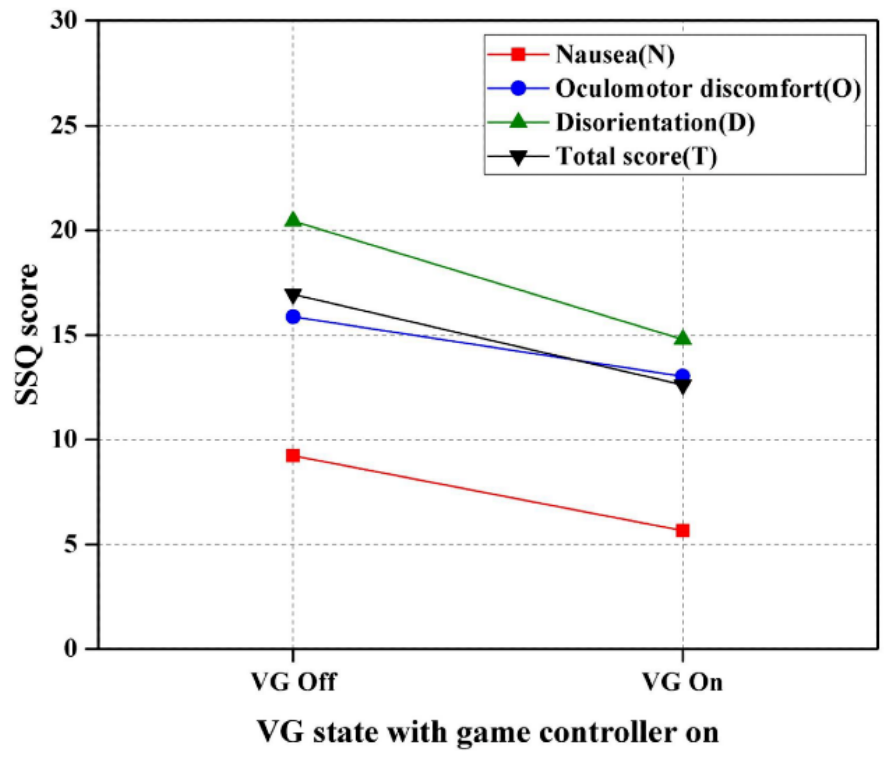

\section{Experiment II: Effect of Visual Guide Size and Position}

In the first step of Experiment II, the paired $t$ test showed that there was a significant difference in VR sickness with the size of visual guide used in S5: nausea and total score values were significantly decreased, whereas oculomotor discomfort and disorientation values had no significant differences. However, there was no significant difference in VR sickness with the size 
of visual guide used in S4 and S6. Table 5 shows the results of the paired $t$ test of the visual guide size with the game controller on. As shown in Figure 9, VR sickness was lower than that observed with other sizes when the visual guide was $30 \%$ of the size of the aspect ratio. Figure 9 shows the results of the first step of Experiment II (ie, difference in VR sickness with respect to the visual guide size when the game controller was on).

In the second step of Experiment II, the paired $t$ test showed that there was a significant difference in VR sickness with the position of visual guide used in S5: nausea and total score values were significantly decreased, whereas oculomotor discomfort and disorientation values had no significant differences. However, there was no significant difference in VR sickness with the position of the visual guide used in S7 and S8. Table 6 shows the results of the paired $t$ test of the visual guide position with the game controller on. As shown in Figure 10, VR sickness was lower than that observed with other positions when visual guide was in the head-tracking direction. Figure 10 shows the results of the second step of Experiment II (ie, difference in VR sickness with respect to visual guide position when the game controller is on).

Table 5. Results of the paired $t$ test of visual guide size with game controller on.

\begin{tabular}{|c|c|c|c|c|c|c|c|c|c|c|c|c|}
\hline \multirow[t]{3}{*}{ Scenario (size) } & \multicolumn{12}{|c|}{ Simulator Sickness Questionnaire } \\
\hline & \multicolumn{3}{|c|}{ Nausea } & \multicolumn{3}{|c|}{ Oculomotor discomfort } & \multicolumn{3}{|c|}{ Disorientation } & \multicolumn{3}{|c|}{ Total score } \\
\hline & Score & $t(d f)$ & $P$ value & Score & $t(d f)$ & $P$ value & Score & $t(d f)$ & $P$ value & Score & $t(d f)$ & $\mathrm{P}$ value \\
\hline $\mathrm{S} 3(0 \%)$ & 9.24 & $\mathrm{~N} / \mathrm{A}^{\mathrm{a}}$ & N/A & 15.87 & $\mathrm{~N} / \mathrm{A}$ & N/A & 20.45 & N/A & N/A & 16.95 & N/A & N/A \\
\hline S4 $(10 \%)$ & 7.45 & $1.063(31)$ & .296 & 12.55 & $1.422(31)$ & .17 & 16.53 & $1.359(31)$ & .18 & 13.56 & $1.674(31)$ & .10 \\
\hline S6 $(50 \%)$ & 7.16 & $0.980(31)$ & .34 & 12.79 & $1.200(31)$ & .24 & 15.23 & $1.615(31)$ & .12 & 13.21 & $1.447(31)$ & .16 \\
\hline
\end{tabular}

${ }^{\mathrm{a}} \mathrm{N} / \mathrm{A}$ : not applicable.

${ }^{\mathrm{b}}$ Italicized values indicate statistical significance.

Figure 9. Results of the first step of experiment II (difference in virtual reality sickness with respect to visual guide size when game controller was on). SSQ: Simulator Sickness Questionnaire; VG: visual guide.

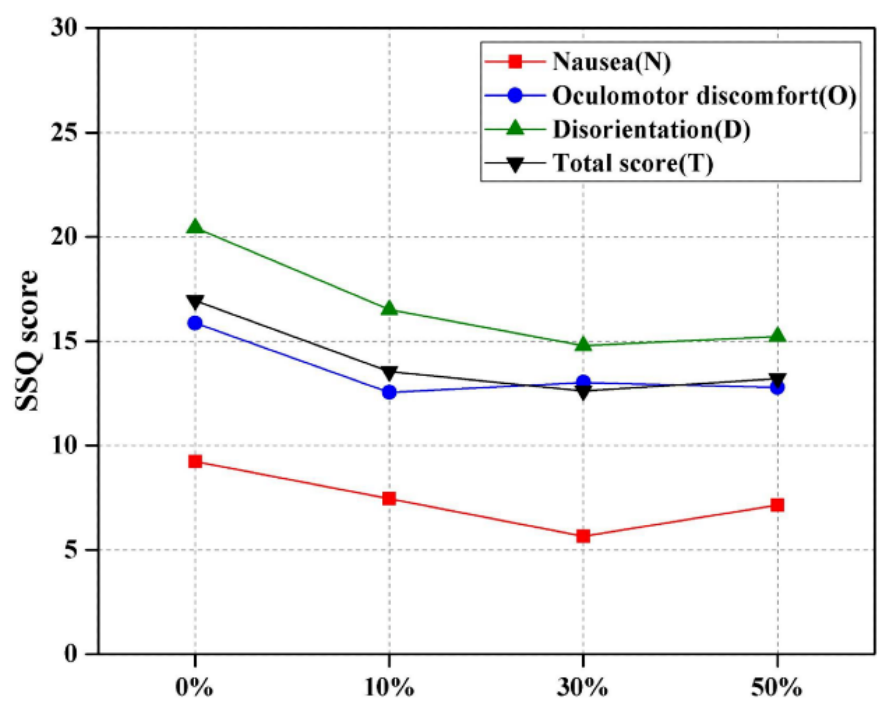

VG size with game controller on 
Table 6. Results of the paired $t$ test of the visual guide position with game controller on.

\begin{tabular}{|c|c|c|c|c|c|c|c|c|c|c|c|c|}
\hline \multirow[t]{3}{*}{ Scenario (position) } & \multicolumn{12}{|c|}{ Simulator Sickness Questionnaire } \\
\hline & \multicolumn{3}{|c|}{ Nausea } & \multicolumn{3}{|c|}{ Oculomotor discomfort } & \multicolumn{3}{|c|}{ Disorientation } & \multicolumn{3}{|c|}{ Total score } \\
\hline & Score & $t(d f)$ & $P$ value & Score & $t(d f)$ & $P$ value & Score & $t(d f)$ & $P$ value & Score & $t(d f)$ & $P$ value \\
\hline S3 (none) & 9.24 & $\mathrm{~N} / \mathrm{A}^{\mathrm{a}}$ & N/A & 15.87 & N/A & N/A & 20.45 & N/A & N/A & 16.95 & N/A & N/A \\
\hline S5 (H) & 5.66 & $2.547(31)$ & $.02^{\mathrm{b}}$ & 13.03 & $1.359(31)$ & 18 & 14.79 & $1.815(31)$ & .08 & 12.62 & $2.183(31)$ & .04 \\
\hline S7 (G) & 9.24 & $0(31)$ & $>.99$ & 18.71 & $-1.099(31)$ & .28 & 18.71 & $0.436(31)$ & .67 & 17.88 & $-0.323(31)$ & .75 \\
\hline S8 (H \& G) & 7.75 & $0.645(31)$ & .52 & 14.69 & $0.456(31)$ & .65 & 19.14 & $0.317(31)$ & .75 & 15.43 & $0.517(31)$ & .61 \\
\hline
\end{tabular}

${ }^{\mathrm{a} N} / \mathrm{A}$ : not applicable.

${ }^{\mathrm{b}}$ Italicized values indicate statistical significance.

Figure 10. Results of the second step of Experiment II (difference in virtual reality sickness with respect to visual guide position when game controller is on). SSQ: Simulator Sickness Questionnaire; VG: visual guide.

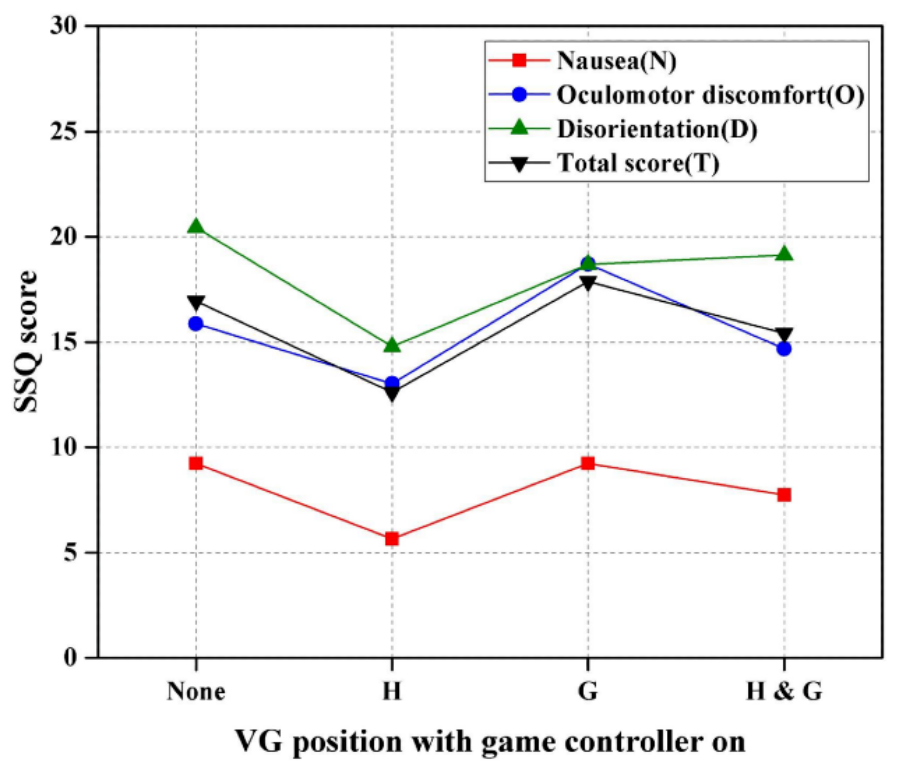

\section{Discussion}

\section{Principal Findings}

In this study, we analyzed the correlation between VR sickness and crosshair, which is widely used as a visual guide in an FPS game. To do this, eight scenarios were designed: visual guide on/off, game controller on/off, and various sizes and positions of visual guide. Experiments were performed using a protocol that consisted of the abovementioned eight scenarios, SSQ, and rest video. Results of Experiment I showed no reduction of VR sickness of the visual guide when the game controller was not operated, whereas there was a reduction when the game controller was operated. While the user operated the HMD and the game controller in the game, the user's gaze movement was synchronized with the motion of the visual guide, thereby reducing VR sickness. The results suggest that visual guide can reduce VR sickness caused by sensory conflicts between content and users when manipulating content. It can be interpreted that visual guide should be used effectively to reduce VR sickness because most VR content requires a game controller. Results of experiment II confirmed a difference in VR sickness with respect to the size and position of the visual guide with game controller operation. VR sickness according to sizes $10 \%$ and
$50 \%$ and positions $\mathrm{G}$ and $\mathrm{H} \& \mathrm{G}$ of the visual guide was not significant, whereas VR sickness according to size $30 \%$ and position $\mathrm{H}$ of the visual guide was significant. Moreover, VR sickness was lower when the visual guide was $30 \%$ of the size of the aspect ratio and positioned in the head-tracking direction compared with other sizes and positions. The various sizes of the visual guide reduced nausea, oculomotor discomfort, disorientation symptom, and total score when compared to scenarios when a visual guide was not used. In particular, the visual guide at $30 \%$ the size of the aspect ratio further reduced these symptoms to a larger extent than other sizes. The visual guide at $50 \%$ of the size of the aspect ratio reduced the disorientation symptom, showing that disorientation was minimized due to the high synergy with the gaze movement when the visual guide size increased. However, if the size of the visual guide increases, the VR fidelity cannot be maintained because it lowers the immersion and presence of the user. The visual guide positioned in the head-tracking direction reduced the symptoms of nausea, oculomotor discomfort, disorientation, and total score more than other positions. As mentioned above, crosshair was the visual guide function to reduce VR sickness, as well as maintain VR fidelity in FPS games. 
When recruiting participants, we tried to keep the number of men and women the same. As a result, the number of male $(n=23)$ participants exceeded the number of female $(n=9)$ participants recruited. This may have affected the results of the experiments, as it is reported that men are stronger than women with regard to motion sickness [29]. Therefore, the effects of the proposed method in a same-gender ratio environment should be included in further study.

\section{Conclusions}

In this study, we used an experimental protocol consisting of scenarios such as visual guide on/off, game controller on/off, and various sizes and positions of the visual guide to analyze the correlation between VR sickness and crosshair that is widely used as a visual guide in FPS games. VR sickness was found to be significantly correlated with visual guide on/off, and the use of a visual guide was very effective in reducing VR sickness when using a game controller. VR sickness was reduced by synchronizing the user's gaze movement to the motion of the visual guide while operating the HMD and game controller within the game. In addition, VR sickness reduced when the visual guide was $30 \%$ of the size of the aspect ratio and positioned in the head-tracking direction. From these findings, it is confirmed that using a visual guide can be an effective method to reduce VR sickness. The experimental results of this study indicate that the visual guide can achieve VR sickness reduction while maintaining user presence and immersion in the virtual environment. In other words, the use of visual guide is expected to solve the existing difficulty in disseminating various VR content due to VR sickness.

\section{Acknowledgments}

This work was supported by Institute for Information \& communications Technology Promotion (IITP) grant funded by the Korea government (Ministry of Science and ICT [MSIT]) (No. 2017-0-00289-001). This work was supported by the National Research Foundation of Korea (NRF) grant funded by the Korea government (MSIT) (No. NRF-2020R1F1A1076114).

In addition, this study was partially supported by the Sabbatical Year Research Program of KOREATECH in 2019.

\section{Conflicts of Interest}

None declared.

\section{References}

1. LaViola JJ. A discussion of cybersickness in virtual environments. SIGCHI Bull 2000 Jan 01;32(1):47-56. [doi: $10.1145 / 333329.333344]$

2. Hettinger LJ, Berbaum KS, Kennedy RS, Dunlap WP, Nolan MD. Vection and simulator sickness. Mil Psychol 1990;2(3):171-181. [doi: 10.1207/s15327876mp0203 4] [Medline: 11537522$]$

3. Bonato F, Bubka A, Palmisano S, Phillip D, Moreno G. Vection change exacerbates simulator sickness in virtual environments. Presence: Teleoperators and Virtual Environments 2008 Jun 01;17(3):283-292. [doi: 10.1162/pres.17.3.283]

4. Palmisano S, Allison RS, Schira MM, Barry RJ. Future challenges for vection research: definitions, functional significance, measures, and neural bases. Front Psychol 2015;6:193 [FREE Full text] [doi: 10.3389/fpsyg.2015.00193] [Medline: 25774143]

5. Keshavarz B, Riecke BE, Hettinger LJ, Campos JL. Vection and visually induced motion sickness: how are they related? Front Psychol 2015;6:472 [FREE Full text] [doi: 10.3389/fpsyg.2015.00472] [Medline: 25941509]

6. Llorach G, Evans A, Blat J. Simulator sickness and presence using HMDs: comparing use of a game controller and a position estimation system. 2014 Nov Presented at: The 20th ACM Symposium on Virtual Reality Software and Technology; 2014; Edinburgh Scotland p. 137-140. [doi: 10.1145/2671015.2671120]

7. Draper MH, Viire ES, Furness TA, Gawron VJ. Effects of image scale and system time delay on simulator sickness within head-coupled virtual environments. Hum Factors 2001;43(1):129-146. [doi: 10.1518/001872001775992552] [Medline: $\underline{11474759}$ ]

8. Duh H, Lin J, Kenyon R, Parker D, Furness T. Effects of field of view on balance in an immersive environment. 2001 Presented at: IEEE Virtual Reality 2001; March 13-17, 2001; Yokohama, Japan p. 235-240. [doi: 10.1109/vr.2001.913791]

9. Shigemasu H, Morita T, Matsuzaki N, Sato T, Harasawa M, Aizawa K. Effects of physical display size and amplitude of oscillation on visually induced motion sickness. 2006 Presented at: The ACM Symposium on Virtual Reality Software and Technology 2006; November 1-3, 2006; Limassol Cyprus p. 372-375. [doi: 10.1145/1180495.1180571]

10. Kim YY, Kim EN, Park MJ, Park KS, Ko HD, Kim HT. The application of biosignal feedback for reducing cybersickness from exposure to a virtual environment. Presence: Teleoperators and Virtual Environments 2008 Feb 01;17(1):1-16. [doi: 10.1162/pres.17.1.1]

11. Bos JE, de Vries SC, van Emmerik ML, Groen EL. The effect of internal and external fields of view on visually induced motion sickness. Appl Ergon 2010 Jul;41(4):516-521. [doi: 10.1016/j.apergo.2009.11.007] [Medline: 20042182]

12. van Emmerik ML, de Vries SC, Bos JE. Internal and external fields of view affect cybersickness. Displays 2011 Oct;32(4):169-174. [doi: 10.1016/j.displa.2010.11.003]

13. Carnegie K, Rhee T. Reducing visual discomfort with HMDs using dynamic depth of field. IEEE Comput Grap Appl 2015 Sep;35(5):34-41. [doi: 10.1109/mcg.2015.98] 
14. Nie G, Duh HB, Liu Y, Wang Y. Analysis on mitigation of visually induced motion sickness by applying dynamical blurring on a user's retina. IEEE Trans Visual Comput Graphics 2020 Aug 1;26(8):2535-2545. [doi: 10.1109/tvcg.2019.2893668]

15. Buhler H, Misztal S, Schild J. Reducing VR sickness through peripheral visual effects. 2018 Presented at: 2018 IEEE Conference on Virtual Reality and 3D User Interfaces (VR); March 18-22, 2018; Tuebingen/Reutlingen, Germany p. 517-519. [doi: 10.1109/vr.2018.8446346]

16. Fernandes A, Feiner S. Combating VR sickness through subtle dynamic field-of-view modification. 2016 Presented at: 2016 IEEE Symposium on 3D User Interfaces (3DUI); March 19-20, 2016; Greenville, SC, USA p. 201-210. [doi: 10.1109/3dui.2016.7460053]

17. Farmani Y, Teather R. Viewpoint snapping to reduce cybersickness in virtual reality. 2018 Presented at: The 44th Graphics Interface Conference; May 8-11, 2018; Toronto Canada p. 168-175. [doi: 10.20380/GI2018.23]

18. Duh HB, Parker DE, Furness TA. An independent visual background reduced simulator sickness in a driving simulator. Presence: Teleoperators \& Virtual Environments 2004 Oct;13(5):578-588. [doi: 10.1162/1054746042545283]

19. Prothero J, Parker D. A unified approach to presence and motion sickness. In: Virtual and adaptive environments: Applications, implications, and human performance issues. Boca Raton, Florida: CRC Press; 2003:47-66.

20. Cao Z, Jerald J, Kopper R. Visually-induced motion sickness reduction via static and dynamic rest frames. 2018 Presented at: 2018 IEEE Conference on Virtual Reality and 3D User Interfaces (VR); March 18-22, 2018; Tuebingen/Reutlingen, Germany p. 105-112. [doi: 10.1109/vr.2018.8446210]

21. Wienrich C, Weidner C, Schatto C, Obremski D, Israel J. A virtual nose as a rest-frame - the impact on simulator sickness and game experience. 2018 Presented at: 2018 10th International Conference on Virtual Worlds and Games for Serious Applications (VS-Games); Sept 5-7, 2018; Würzburg, Germany p. 1-8. [doi: 10.1109/vs-games.2018.8493408]

22. Duh HL, Parker DE, Furness TA. An "independent visual background” reduced balance disturbance envoked by visual scene motion: implication for alleviating simulator sickness. 2001 Presented at: The SIGCHI Conference on Human Factors in Computing Systems; March 31 - April 5, 2001; Seattle Washington USA p. 85-89. [doi: 10.1145/365024.365051]

23. Lin JJ, Abi-Rached H, Kim D, Parker DE, Furness TA. A “natural” independent visual background reduced simulator sickness. Proceedings of the Human Factors and Ergonomics Society Annual Meeting 2002 Sep 1;46(26):2124-2128. [doi: $10.1177 / 154193120204602605]$

24. Prothero J. The role of rest frames in vection, presence and motion sickness. Doctoral Dissertation. University of Washington. Seattle, Washington: University of Washington; 1998.

25. Rosenblatt M. A central limit theorem and a strong mixing condition. Proc Natl Acad Sci U S A 1956 Jan;42(1):43-47 [FREE Full text] [doi: 10.1073/pnas.42.1.43] [Medline: 16589813]

26. Kennedy RS, Lane NE, Berbaum KS, Lilienthal MG. Simulator sickness questionnaire: An enhanced method for quantifying simulator sickness. Int J Aviat Psychol 1993 Jul;3(3):203-220. [doi: 10.1207/s15327108ijap0303 3]

27. Kennedy R, Drexler J, Compton D, Stanney K, Lanham D, Harm D. Configural scoring of simulator sickness, cybersickness and space adaptation syndrome: similarities and differences. In: Virtual and adaptive environments: applications, implications, and human performance issues. Boca Raton, Florida: CRC Press; 2003:247.

28. Häkkinen J, Ohta F, Kawai T. Time course of sickness symptoms with HMD viewing of 360-degree Videos. Electronic Imaging 2019 Jan 13;2019(3):60403-1-60403-11. [doi: 10.2352/j.imagingsci.technol.2018.62.6.060403]

29. Stanney KM, Kennedy RS, Drexler JM, Harm DL. Motion sickness and proprioceptive aftereffects following virtual environment exposure. Applied Ergonomics 1999 Feb;30(1):27-38. [doi: 10.1016/s0003-6870(98)00039-8]

\section{Abbreviations}

FPS: first-person shooter

G: movement direction with game controller

H: head-tracking direction with head-mounted display

H \& G: head-tracking direction with head-mounted display and movement direction with game controller

HMD: head-mounted display

IITP: Institute for Information \& Communications Technology Promotion

IVB: independent visual background

MSIT: Ministry of Science and ICT

SSQ: Simulator Sickness Questionnaire

VR: virtual reality 
Edited by N Zary; submitted 29.01.20; peer-reviewed by C Johnson, $R$ Haghighi Osgouei; comments to author 30.06.20; revised version received 25.03.21; accepted 30.04.21; published 15.07.21

Please cite as:

Seok KH, Kim Y, Son W, Kim YS

Using Visual Guides to Reduce Virtual Reality Sickness in First-Person Shooter Games: Correlation Analysis

JMIR Serious Games 2021;9(3):e18020

URL: https://games.jmir.org/2021/3/e18020

doi: $10.2196 / 18020$

PMID: $\underline{34264196}$

CKwang-Ho Seok, YeolHo Kim, Wookho Son, Yoon Sang Kim. Originally published in JMIR Serious Games (https://games.jmir.org), 15.07.2021. This is an open-access article distributed under the terms of the Creative Commons Attribution License (https://creativecommons.org/licenses/by/4.0/), which permits unrestricted use, distribution, and reproduction in any medium, provided the original work, first published in JMIR Serious Games, is properly cited. The complete bibliographic information, a link to the original publication on https://games.jmir.org, as well as this copyright and license information must be included. 